\title{
Procesos operativos para la admisión estudiantil en universidades públicas de la Costa Oriental del Lago
}

\author{
Operational processes for student admission to public universities on the \\ East Coast of Lake
}

$\begin{array}{lr}\text { Eglys Rodríguez } & \text { Artículo recibido en febrero } 2019 \\ \text { eglysdvrg@gmail.com } & \text { Arbitrado en marzo } 2019 \\ \text { Código ORCID: oooo-0oo2-3076-0719 } & \text { Publicado en mayo } 2019 \\ \text { Universidad del Zulia, Venezuela } & \end{array}$

\section{Resumen}

La investigación tuvo como propósito describir el proceso operativo aplicado en los departamentos de admisión estudiantil de las universidades públicas de la Costa Oriental del Lago. El estudio fue descriptivo con diseño de campo, no experimental, transeccional. La población estuvo conformada por las universidades públicas de la Costa Oriental del Lago. La técnica de recolección fue la encuesta y el instrumento un cuestionario con escala de frecuencia, compuesto por 15 ítems, se utilizó el juicio de expertos para su validez y el coeficiente Alfa Cronbach para su confiabilidad, obteniéndose 0,96. El análisis de los datos se realizó mediante la estadística descriptiva, a través del estudio de la media aritmética. Se concluye que se aplican planes de acciones que permiten planificar y controlar los servicios ofrecidos para alcanzar los objetivos operativos, a través de la decisión del proceso, diseño del proceso, control del proceso, mejoras del proceso y selección del proceso.

\footnotetext{
Abstract

The purpose of the research was to describe the operational process applied in the student admission departments of the public universities of the Eastern Coast of the Lake. The study was descriptive with a field design, not experimental, transectional. The population was conformed by the public universities of the Eastern Coast of the Lake. The collection technique was the survey and the instrument was a questionnaire with a frequency scale, made up of 15 items. Expert judgment was determined for its validity and the Alpha Cronbach coefficient for its reliability, obtaining 0.96 . The data analysis was carried out using descriptive statistics, through the study of the arithmetic mean. It is concluded that action plans are required that allow controlling and controlling the services received to achieve the operational objectives, through the decision of the process, process design, process control, process improvements and process selection.
}

Palabras clave:

Control del proceso; decisión del proceso; diseño del proceso; proceso operativo; selección del proceso
Keywords:

Process control; process decision; process design; operational process; process selection 
INTRODUCCIÓN

Durante muchos años, la búsqueda de la calidad en los procesos y servicios ejecutados por una empresa ha sido el norte esencial en su constante accionar. De hecho, la calidad implica mejorar permanentemente la eficacia y eficiencia de la organización en cuanto a sus actividades, y estar siempre muy atento a las necesidades del cliente (haciendo énfasis en las quejas o muestras de insatisfacción).

En tal sentido, es evidente que si se planifican, depuran y controlan los procesos de trabajo, ocurrirá un aumento en la capacidad de la organización, al igual que su rendimiento. Pero, además, es necesario indagar con cierta regularidad sobre la calidad que percibe el cliente y las posibilidades tangibles de mejorar el servicio recibido. La misma, se encuentra condicionada por la forma en que la organización realiza todas las actividades que repercuten en el servicio que presta a sus clientes.

La situación anteriormente expuesta, plantea la existencia de un entorno donde los cambios se suscitan con mayor rapidez, los competidores mejoran continuamente los productos y servicios ofertados, los avances tecnológicos conducen a la obtención de productos sustitutivos y los valores, costumbres y hábitos del consumidor también cambian, haciendo evolucionar las necesidades de los clientes. Entonces, la organización debe identificar en qué mercado está actuando y cuáles son las expectativas de los clientes que tiene respecto a los atributos del servicio que está contratando.

De esta forma, la dirección debe dotar a la organización de una estructura que permita cumplir con la misión (objetivo primordial) y la visión (percepción del cliente sobre el servicio prestado) establecidas. En tal sentido, la implantación de la gestión de procesos se ha revelado como una de las herramientas de mejora de la gestión más efectivas para todos los tipos de organizaciones.

Lo anteriormente señalado tiene su sustento en lo expuesto por Bravo (2011), quien define la gestión por procesos como una disciplina que ayuda a la dirección de la empresa a identificar, representar, diseñar, formalizar, controlar, mejorar y hacer más productivos los procesos de la organización para lograr la confianza del cliente, logrando así mejorar su calidad de servicios.

Visto así, la gestión por procesos es una forma de organización diferente de la clásica organización funcional, ya que los procesos son gestionados de modo estructurado y sobre su mejora se basa la propia organización. Su objetivo se centra en los distintos aspectos de cada proceso: qué se hace (cuál es el proceso y quien es la persona o personas responsables), para quién (quiénes son los clientes externos o internos del proceso) y cómo deben ser los resultados del proceso para adecuarse a las necesidades de los clientes internos, a fin de mejorar las operaciones.

De manera que, cualquier actividad o conjunto de actividades ligadas entre sí, que utiliza recursos y controles para transformar elementos de entrada en resultados, puede considerarse como un proceso. Asimismo, todas las actividades de la organización, desde la planificación de las compras hasta la atención de un reclamo, pueden y deben considerarse entonces como procesos. 
Así pues, la identificación y gestión sistemática de los procesos que se realizan en la organización, y en particular las interacciones entre tales procesos, se conoce como enfoque basado en procesos. Dicho enfoque de gestión no va dirigido a la detección de errores en el servicio; sino a la forma de concebir cada proceso, esto es lo que ha de permitir evaluar las desviaciones del mismo a fin de corregir la tendencia actual, antes de que se produzca un resultado defectuoso.

En este orden de ideas, se destaca el proceso operativo, el mismo según criterio de Fernández (2010), combinan y transforman recursos acorde con las exigencias de los clientes para lograr el producto o proporcionar el servicio deseado. Por consiguiente, este tipo de proceso dentro de una organización, es capaz de reaccionar autónomamente a los cambios, mediante un control constante de la capacidad de cada proceso, la mejora continua, flexibilidad estructural y la orientación de las actividades hacia la plena satisfacción del cliente $\mathrm{y}$ de sus necesidades.

Este es uno de los mecanismos más efectivos para que la organización alcance unos altos niveles de eficiencia. Para adoptar un enfoque basado en procesos, la organización debe identificar todas y cada una de las actividades que realiza: en dicha actividad debería intervenir toda la organización, a través de un equipo multidisciplinar con presencia de personas conocedoras de los diferentes procesos.

Ahora bien, en el caso de América Latina, según Mallar (2010), empresas líderes aplicaron el cambio organizativo, individualizando sus procesos, eligiendo los procesos relevantes, analizándolos y mejorándolos y finalmente utilizando este enfoque para transformar sus organizaciones. Luego de los buenos resultados logrados, aplicaron la experiencia obtenida para optimizar el resto de sus procesos en toda la organización.

De esta manera, en el contexto empresarial surge la concepción de proceso estratégico como uno de los elementos esenciales de la administración exitosa de las organizaciones de principios del siglo XXI (Mariño, 2001). Para ello las empresas deben tener claro lo que implica gestionar toda la organización basándose en los procesos, siendo definidos éstos como una secuencia de actividades orientadas a generar un valor añadido sobre una entrada para conseguir un resultado, y una salida que a su vez satisfaga los requerimientos del cliente (Rojas, 2003).

Ante este contexto, no escapan las universidades públicas, las cuales en su búsqueda de formar nuevos profesionales deben establecer estrategias que les permitan captar los jóvenes con deseos de formación en tercer nivel. Al respecto Serrano (2003) plantea que las instituciones universitarias pueden ser vistas como sistemas complejos abiertos, que funcionan dentro de las condiciones de contextos específicos en las sociedades concretas, en donde interactúan una serie de procesos para dar como resultado un producto y/o un servicio.

Ahora bien, vale asentar que uno de los procesos que requiere de una gestión está referido a los de admisión estudiantil, como un proceso sistémico de la política matricular de las instituciones universitarias, concebida en tres componentes fundamentales: información, tamaño de la matrícula y la permanencia 
estudiantil. En tal sentido, Guevara et al. (2011) refieren que el proceso de cambio que vive actualmente el sistema de ingreso universitario de Venezuela, plantea a las universidades la necesidad de revisar los mecanismos que le permitan conocer el impacto que tiene el nuevo proceso que se ha venido implementando desde el Consejo Nacional de Universidades (CNU).

En virtud de ello, las instituciones universitarias deben realizar esfuerzos mancomunados que les permitan llevar a cabo transformaciones, introduciendo adelantos e innovaciones que respondan de manera eficaz a los progresos y cambios que definen esta época, asumiendo este reto como estrategia para consolidar las universidades. En el caso específico de las universidades públicas de la Costa Oriental del Lago, el proceso de admisión se centra en los fundamentos legales de la educación universitaria venezolana, cuyo fin principal es la inserción de jóvenes según sea el Plan de Crecimiento Matricular de cada institución.

Es de hacer notar que, a través de un diagnóstico previo realizado por la investigadora se pudo evidenciar que en los departamentos de admisión estudiantil la función principal es la de asesorar, coordinar y supervisar la administración o funcionamiento del proceso de matrícula en las carreras ofertadas por la universidad, con el propósito de mantener la uniformidad del proceso, desde el punto de vista de la aplicación de normas y procedimientos, del uso y manejo de la documentación, materiales, junto a la recolección y procesamiento de la información generada en el proceso. De esta manera, se garantiza un alto grado de confianza en el éxito, así como en el cumplimiento de los requisitos exigidos por el Consejo Nacional de Universidades (CNU).

Sin embargo, aun cuando en estas instituciones se ha venido observando un mayor reconocimiento de la importancia que tiene lograr una efectiva gestión para alcanzar un control eficiente del proceso de admisión estudiantil, según la percepción del personal que labora en la misma, existen deficiencias, lo cual ha generado debilidades en todo el proceso, ya que tanto la información como las operaciones no fluyen correctamente.

En específico, en entrevistas realizadas a los coordinadores académicos y secretarios docentes, se señalan la improvisación en el momento de establecer el cronograma de asignación de documentos, falta de personal de apoyo que oriente a los nuevos ingresos, carencia de tecnología de vanguardia disponible para los aspirantes de escasos recursos que requieren procesar su inscripción vía web, la asignación masiva en algunas carreras o programas académicos y la poca en otras.

Bajo esta óptica, se evidenció la conveniencia de realizar un análisis de la gestión por procesos y de allí se realiza este artículo cuyo propósito es describir el proceso operativo aplicado en los departamentos de admisión estudiantil de las universidades públicas de la Costa Oriental del Lago.

\section{Procesos operativos}

La gestión por procesos es un conjunto de procedimientos o esquemas que permite organizar los esfuerzos y la utilización de los recursos para lograr la satisfacción de aquello que está vinculado con los procesos que definen al sistema 
organizacional. Dichos procedimientos o esquemas requieren que todas las partes que lo componen, se caractericen por crear relaciones coordinadas, para lograr niveles de eficacia, eficiencia y efectividad, que cumplan con los elementos básicos de la gestión de calidad y productividad que es alcanzar los requerimientos de los clientes en los tiempos establecidos y a los costos presupuestados. Estos procedimientos se dividen en estratégicos, operativos o clave y de apoyo tal como se describe en la figura 1.

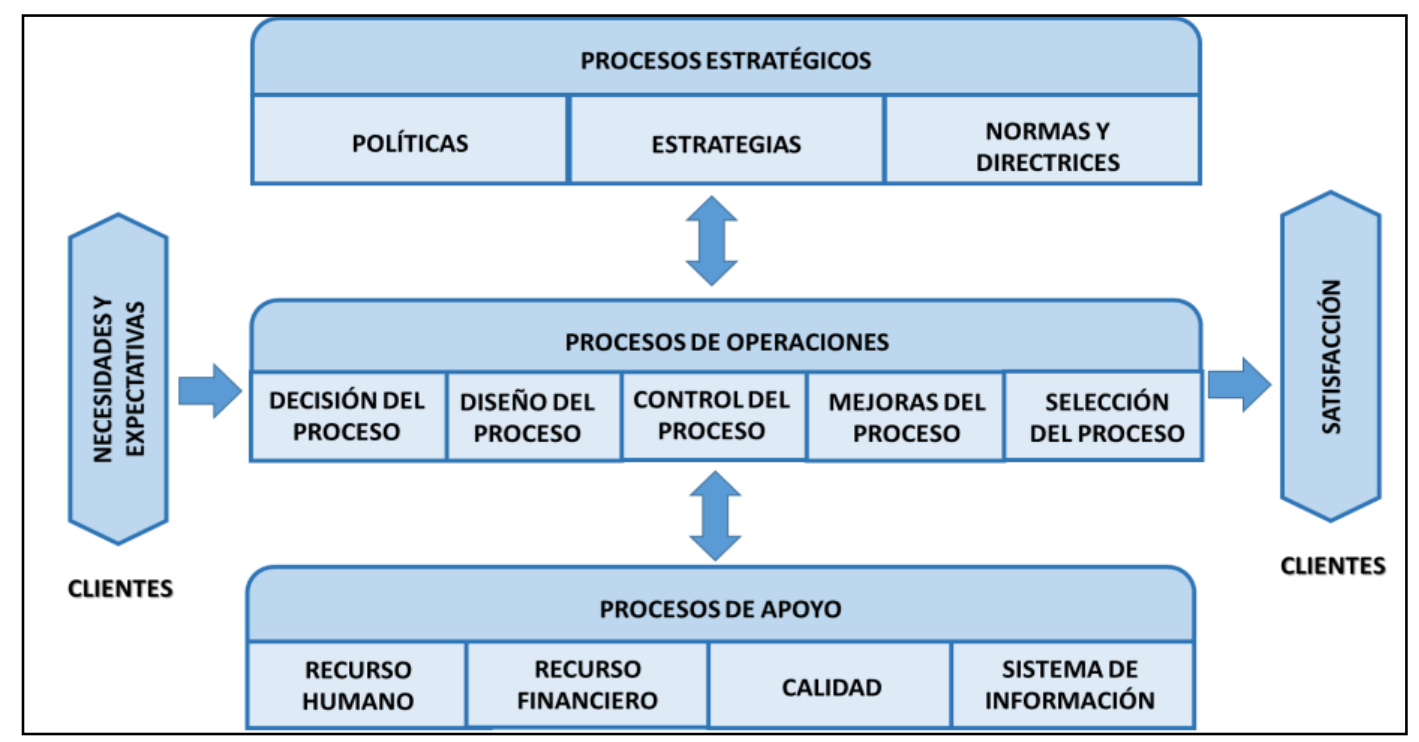

Figura 1. Tipos de proceso. (Fuente: Adaptación propia)

En la perspectiva de Mallar (2010), los procesos operativos son aquellos que afectan la satisfacción del cliente y la misión de la organización de forma directa, representan una actividad primaria en la cadena de producción de valor. Por su parte, Arnoletto (2010) señala que los procesos de operaciones van fijados a la estrategia de operaciones de la empresa, en busca de constituirse en un plan de acción a largo plazo para la producción de los bienes y servicios de la empresa, incluye objetivos operativos de largo plazo, estrategias operativas, distribución de recursos entre los productos $\mathrm{y} / \mathrm{o}$ funciones $\mathrm{y}$ criterios para tomar decisiones.
$\mathrm{Al}$ respecto, Skinner (2010) indica que el proceso de operaciones se divide en dos funciones básicas, la primera configurar un marco de referencia para planificar y controlar la producción, y como segunda fijar las pautas para evaluar el aporte de la gestión de operaciones a los objetivos generales de la empresa. Cervera (2002) lo define como los fundamentales para la obtención del producto, son los dueños del conocimiento de la organización, en conclusión generan y aportan valor, y el cliente es el inicio y el fin del ciclo.

En el mismo orden de ideas, Mariño (2001) los define como esenciales, esto se debe a que indican las actividades primarias, aportan gran valor añadido y 
necesitan recursos para ejecutarse. Representan el plan de acción para alcanzar los objetivos operativos y conseguir la misión de la organización en conjunto y de esa forma crear una ventaja competitiva.

En resumen el proceso de operaciones depende de la dirección o impulso general para la toma de decisiones, debe dar como resultado un patrón consistente $\mathrm{y}$ una ventaja competitiva para la compañía. Establecen criterios y métodos que aseguren de manera eficaz las actividades que comprenden, lo que involucra, centrarse en actividades operacionales y en las características relevantes que permitan el control y la gestión del proceso (Rodríguez, 2008).

Con base a los señalamientos expuestos por los autores, la investigadora concluyó que los procesos operativos también llamados claves son los responsables en añadir el valor agregado en el producto o servicio y es necesario identificar los procesos críticos en los que se debe sobresalir con excelencia, para satisfacer los objetivos de la organización y las expectativas de los clientes. Por consiguiente, el proceso de operaciones será abordado mediante la decisión, diseño, control, mejoras y selección del proceso, los cuales representan indicadores válidos para estudiar esta dimensión.

\section{Decisión de proceso}

La decisión del proceso, según Heizer y Render (2009) es el método que utiliza la organización para convertir los recursos en bienes y servicios, sean estos procesos de oficinas, proveedores de servicios o fabricantes, los gerentes de operaciones deben tomar en consideración decisiones como la selección del proceso, integración vertical, flexibilidad de recursos, participación del cliente e intensidad del capital, por lo cual es necesario analizar, diseñar controlar y mejorar continuamente los procesos de las empresas.

De igual modo, la gerencia debe analizar y optimizar la interacción de los procesos, para lo que se debe asegurar de forma eficaz y eficiente la secuencia de los procesos diseñados con tal fin, para lograr los resultados deseados en los planes empresariales. En la opinión de Heizer y Render (2009) al analizar y diseñar procesos para transformar recursos en bienes y servicios, es necesario realizar preguntas como: ¿Está diseñado el proceso, en términos de singularización, respuesta o bajo costo, para alcanzar ventajas competitivas? ¿Los pasos que no añaden valor están siendo eliminados? ¿Se logra maximizar el valor ofrecido al cliente desde su punto de vista? ¿Conseguirá pedidos el proceso?

En el mismo orden de ideas, Krajewski y Ritzman (2008) se refieren a la decisión del proceso como un paso esencial y necesario de usar en la elaboración de productos o en el suministro de servicios. Tomar una decisión acerca de los procesos incluye opciones diferentes en relación a la selección de recursos humanos, equipos y materiales. Las decisiones de proceso afectan los logros de la empresa con respecto a las prioridades competitivas de calidad, flexibilidad, tiempo y costo, debido a que son estrategias que afectan la capacidad de una organización para competir en un largo periodo de tiempo.

En este particular, la investigadora concluye que la decisión del proceso direcciona a la elección de una secuencia 
de actividades que requieren recursos, agregan valor y utilizan controles para ser orientados a producir un valor agregado sobre una entrada para conseguir un resultado, y una salida que satisfaga las exigencias del cliente, con el fin de permitir la transformación de las entradas en las salidas, y así medir la eficiencia del proceso y los resultados esperados.

\section{Diseño del proceso}

Después de identificar las actividades y acciones sistemáticas que se realizan de forma definida y va dirigida al logro de un objetivo definido, se elabora y validan los procesos con sus diagramas de flujo y gráficos, que permiten investigar los procesos con el fin de identificar posibles áreas de mejora, de manera teóricopráctica (Heizer y Render, 2009).

Para Krajewski y Ritzman (2008) es necesario encontrar una decisión del proceso a utilizar, de forma tal que se produzcan bienes que llenen las expectativas de los clientes, las especificaciones del producto dentro de costo y otras restricciones administrativas, el objetivo del diseño de proceso. Todo esto se da luego de idealizar los procesos, definir y seleccionar los pasos a seguir para ejecutar actividades que interactúan, para transformar los elementos de entrada en resultados para minimizar tiempo y maximizar beneficios.

A criterio de la investigadora, luego de tener una idea sobre cuáles son los procesos que se deben implementar, es necesario definir y seleccionar que pasos se deben llevar a cabo para ejecutar las actividades que le darán cumplimiento al proceso para convertir las entradas en resultados, estructurando en forma tal que se logre minimizar tiempos y maximizar beneficios.

\section{Control de proceso}

Una de la fases más importantes en la administración es el control, porque es el mecanismo que garantiza e informa si los hechos van de acuerdo con los objetivos, si no existe control el ejecutivo no podrá comprobar cuál es la situación real de la empresa aunque la misma cuente con magníficos planes, una estructura organizacional adecuada y una dirección eficiente.

Según Slack et al. (2007) lo definen como un mecanismo que a través de indicadores cualitativos y cuantitativos permite corregir desviaciones dentro de un contexto amplio, para cumplir los objetivos claves para el éxito organizacionales le conoce como un proceso informal donde se evalúan factores culturales, organizativos, humanos y grupales.

Siguiendo el orden de las ideas, Heizer y Render (2009) definen el control como la técnica que sirve para comprobar o vigilar lo que se está haciendo y de este modo asegurar que el trabajo en forma satisfactoria hacia el objetivo predeterminado. Por su parte Galván (2009) afirma que los controles son un sistema formado por indicadores, sus objetivos y cuadros de mando resultantes para la toma de decisiones, y sirve para evaluar la marcha del proceso, corregir deficiencias y mejorar continuamente. Por otro lado, para Ruiz (2013) el control es la consecuencia lógica de administrar, es una función indelegable de todo cargo con responsabilidad de conducción.

El control es el sistema que facilita información y detecta oportunidades para poder decidir, con él se logra dominar situaciones, evaluando resultados, corrigiendo la acción para lograr los objetivos esperados en cada proceso. 
Según Pérez (2010) lo define como la función administrativa en todo proceso con la que se puede evaluar el rendimiento y todas las actividades que se inician para garantizar que las operaciones concuerden con las operaciones planificadas.

Por su parte, Rey (2013) indica que el control del proceso se divide en varios tipos pero enfatiza en dos que son el análisis de tareas del proceso que es centrado en las actividades humanas y el análisis de productos del proceso que va orientado a las actividades que se le realiza al objeto.

En opinión de la investigadora, el control del proceso tiene la finalidad de recolectar datos para realizarles algún tipo de mejora, logrando elevar la calidad del proceso, aumentar la eficiencia y reducir costos. Es obligación de los gerentes ser garantes de este control por medio de evaluaciones, tomando las medidas necesarias para reducir las ineficiencias.

\section{Mejoras del proceso}

De acuerdo a los señalamientos de Heizer y Render (2009), se habla de mejora de procesos cuando se optimiza la efectividad y eficiencia del sistema, reforzando mecanismos y controles, para dar respuesta a las contingencias y demandas de los clientes. Para la mejora de los procesos es de considerar: el análisis de los flujos de trabajo, la fijación de objetivos que cumplan con los requerimientos de los clientes, el desarrollo de actividades de mejora y la responsabilidad de los actores del proceso.

Refieren los autores citados, solo puede existir mejora de procesos si los integrantes de la organización se esfuerzan y comprometen en hacer el trabajo bien, para lo que se necesita asignar responsables que se encarguen de los procesos, la documentación, los requisitos de los proveedores, requisitos y necesidades de los clientes internos, de requisitos, expectativas y grado de satisfacción de los clientes externos, indicadores, criterios de medición y herramientas de mejora estadística.

Es necesario que el responsable de cada área sepa que se debe mejorar, para poder realizar una mejora continua del proceso, basado en el cumplimiento o no de los objetivos de la empresa. Para dicha mejora se realizaría la siguiente secuencia de actividades:

$\checkmark$ Definición del problema o desviación detectada sobre los objetivos e indicadores.

$\checkmark$ Establecimiento de los mecanismos de medición.

$\checkmark$ Identificación de las causas, a través del análisis de los datos, resaltando la más relevante, estableciendo posibles soluciones para tomar la más adecuada.

$\checkmark$ Establecimiento de planes de acción e implementar la mejora.

$\checkmark$ Control de la mejora del proceso, monitoreándolos constantemente.

Por su parte, Bravo (2011) opina que quizás por los cambios en la tecnología y los deseos y exigencias de los clientes, lo que fue una actividad fundamental en el pasado en la actualidad ya no lo sean, al identificar todas las actividades adicionales al mapa de procesos, se visualiza cuales podrán ser desechadas para lograr las mejoras.

En conclusión, a criterio de la investigadora, la mejor forma de ahorrar tiempo, dinero y esfuerzo es eliminando las actividades que no agregan valor con 
metodologías de mejora del proceso, ya que distraen del objetivo principal y son dispendiosas, al eliminarlas se clarifica el proceso para enfocarse en las necesidades, requerimientos y expectativas del cliente.

\section{Selección de proceso}

En lo que concierne a la selección del proceso, Chase et al. (2009) afirman que la gestión por proceso hace énfasis en que la forma más eficiente de obtener los resultados es considerando las actividades agrupadas entre sí, para que permitan la transformación de entras y salidas aportando valor al tiempo que se ejerce control. En su opinión la selección de proceso se debe tener presente que se ha clasificado en dos dimensiones: según el flujo del producto y el tipo de fabricación.

Cada producto debe elaborarse mediante uno de estos procesos, sin embargo puede existir la combinación de ambos, es común encontrarse con varios tipos de procesos operacionales dentro de la misma planta física. Es notorio que estos procesos son aplicables a empresas que prestan servicios como a empresas que producen bienes, como lo explican Heizer y Rendel (2009) siempre y cuando se tomen en cuenta ciertos factores como: condiciones de mercado, necesidades de capital, mano de obra, habilidades gerenciales, materia prima utilizada $\mathrm{y}$ tecnología.

Según Kaplan y Norton (2009) se deben analizar los procesos internos, en aras de conseguir la satisfacción total del cliente y altos niveles de rendimiento financiero, con la finalidad de determinar las áreas críticas y aplicar los correctivos necesarios para detectar la selección de procesos que mejor se adapte a la actividad.
Es opinión de la investigadora, que es función de los directivos identificar los procesos críticos en los que se requiere sobresalir con excelencia, satisfaciendo los objetivos de los accionistas y los clientes a través de la selección de procesos, asumiendo las teorías de Chase et al. (2009) que clasifica la selección de procesos según el flujo del producto y el tipo de fabricación.

Para Schroeder (2007), el flujo del producto asociado al tipo de fabricación se divide en tres tipos: lineal, intermitente y por proyecto. En el flujo lineal encontramos una secuencia de operaciones lineales para fabricar el producto y se divide en masiva o en masa y continúa.

Siguiendo el orden de ideas, Kaplan y Norton (2009) afirman que pasar de los años y el avance de la tecnología han hecho posible una flexibilidad sustancial en las líneas de ensamble, gracias al uso de control computarizado y la reducción de los tiempos innecesarios, estas solo justifican un número limitado de situaciones, ya que es necesario un alto volumen y productos o familia de productos estandarizados.

Por otro lado, el flujo intermitente es caracterizado por producir lotes en intervalos intermitentes, donde se organizan la mano de obra y los equipos en estaciones de trabajo, lo que hace las operaciones intermitentes estrechamente flexibles para cambiar el producto o el volumen, ya que el producto solo pasa por los centros de trabajo necesarios con equipos de propósitos generales y mano de obra calificada.

Schroeder (2007) indica que la característica más resaltante de los procesos intermitentes es que agrupan 
equipos similares y habilidades de trabajo parecidas, justifican la falta de estandarización o el volumen bajo, es la más económica y la de menor riesgo mientras que los de flujo lineal lujo lineal son llamados distribución por productos debido a que los procesos, el equipo y las habilidades laborales están en de forma secuencial.

Cuando el referido autor, habla de flujo por proyecto, se refiere a la elaboración de productos únicos, es decir, cada unidad representa un producto, no existe un flujo del producto para un proyecto, pero si una secuencia de operaciones y es utilizada cuando hay gran necesidad de creatividad y conceptos únicos, las operaciones individuales son colocadas secuencialmente para lograr los objetivos definitivos del proyecto.

En la opinión de la investigadora, la selección del proceso está determinada por el tipo de proceso que será utilizado para prestar el servicio o fabricar el producto, por lo tanto es necesario tomar en cuenta el volumen y la estandarización del producto, ya que las mismas son de naturaleza estratégica y necesita un perspectiva que resulte efectiva a largo plazo trabajando de forma coordinada en las diferentes unidades de la empresa.

\section{MÉTODO}

El estudio se tipificó como descriptivo con un diseño de campo, no experimental y transeccional. La población estuvo conformada por las universidades públicas de la Costa Oriental del Lago, siendo estas la Universidad del Zulia (LUZ), la Universidad Experimental Rafael María Baralt (UNERMB), y el Instituto Universitario de Tecnología (IUTC), cuyos sujetos informantes fueron 29 personas responsables del ingreso estudiantil en estas instituciones: 2 coordinadores docente, 5 secretarios docentes, y 29 trabajadores del personal administrativo.

La técnica de recolección de datos utilizada fue la encuesta y el instrumento un cuestionario con escala de frecuencia de cinco opciones de respuestas que van desde Siempre (5), Casi Siempre (4), Algunas veces (3), Casi nunca (2) y Nunca (1), compuesto por 15 ítems, se utilizó el juicio de expertos para la validez del mismo y el coeficiente Alfa Cronbach para su confiabilidad, obteniéndose un valor de 0,96. El análisis de los datos se realizó mediante la estadística descriptiva, a través del estudio de la media aritmética, para tal efecto, la investigadora diseñó un baremo para el análisis, tal como se refleja en el cuadro 1.

Cuadro 1. Baremo para la interpretación de la media aritmética

\begin{tabular}{cccc}
\hline Alternativas & Opción de respuesta & Rango para la media & $\begin{array}{c}\text { Nivel de respuesta } \\
\text { para la variable }\end{array}$ \\
\hline $\mathbf{5}$ & Siempre(S) & $4.20 \leq 5.00$ & Muy alta aplicación \\
$\mathbf{4}$ & Casi Siempre (CS) & $3.40<4.20$ & Alta aplicación \\
$\mathbf{3}$ & Algunas veces (AV) & $2.60<3.40$ & Moderada aplicación \\
$\mathbf{2}$ & Casi nunca (CN) & $1.80<2.60$ & Baja aplicación \\
$\mathbf{1}$ & Nunca(N) & $1.00<1.80$ & Muy baja aplicación \\
\hline
\end{tabular}

Fuente: Elaboración propia. 
RESULTADOS

A efectos de iniciar el análisis, se presentan los resultados obtenidos para cada uno de los indicadores que conforman la dimensión proceso estratégico. Así las cosas, en la Tabla 1 se presentan un resumen del indicador decisión del proceso, observándose una media aritmética para el indicador de 4,17 indicando que la decisión del proceso, como parte del proceso de operaciones, llega a una categoría de muy alta aplicación, según el baremo diseñado.

Tabla 1. Indicador: Decisión del proceso

\begin{tabular}{|c|c|c|}
\hline Ítem & $\square$ & Categorías \\
\hline $\begin{array}{l}\text { 1. Analizan la interacción de sus procesos, } \\
\text { asegurándose de que la secuencia de los mismos } \\
\text { se diseñe con el fin de lograr los resultados } \\
\text { deseados. }\end{array}$ & 4,21 & Muy alta aplicación \\
\hline $\begin{array}{l}\text { 2. Al momento de diseñar los procesos se visualiza } \\
\text { el alcance de ventajas competitivas. }\end{array}$ & 4,00 & Alta aplicación \\
\hline \multirow[t]{2}{*}{$\begin{array}{l}\text { 3. Examinan cada paso de sus procesos buscando la } \\
\text { forma de responder con mayor rapidez a los } \\
\text { usuarios. }\end{array}$} & 4,31 & Muy alta aplicación \\
\hline & 4,17 & Alta aplicación \\
\hline
\end{tabular}

Fuente: Elaboración propia.

Lo anteriormente descrito se enmarca atendiendo a los valores individuales de cada ítem, al considerar los encuestados con muy alta aplicación que la dirección se asegura de que la secuencia de sus procesos se diseñen con el fin de lograr los resultados deseados, y que examinan cada paso de sus procesos buscando la forma de responder con mayor rapidez a los usuarios; no obstante con alta aplicación se visualiza el alcance de ventajas competitivas.

Tomando en cuenta los resultados de cada ítem, se determina la decisión del proceso como un proceso operativo en los departamentos de admisión estudiantil de las universidades analizadas, el cual permite analizar y optimizar la interacción de los procesos de admisión para obtener las metas deseadas, por quienes realizan la gestión por procesos.

Estos hallazgos, validan los postulados de Krajewski y Ritzman (2008), quienes refieren que la decisión del proceso es un paso esencial y necesario e incluye opciones diferentes en relación a la selección de recursos humanos, equipos y materiales. Estas decisiones afectan los logros de la empresa con respecto a las prioridades competitivas de calidad, flexibilidad, tiempo y costo, debido a que son estrategias que afectan la capacidad de una organización para competir en un largo periodo de tiempo. De igual manera, existe concordancia con la posición de la investigadora, quien considera que la decisión del proceso direcciona a la elección de una secuencia de actividades que requieren recursos, agregan valor y utilizan controles para conseguir la satisfacción del usuario.

En la Tabla 2 se resumen los resultados obtenidos para el indicador diseño del proceso, destacándose un 
promedio de 4,17, e indica una alta aplicación de las actividades que los departamentos de admisión estudiantil aplican. Así las cosas, las universidades bajo estudio, al enfrentar los retos en el contexto de la admisión estudiantil, identifican las actividades y acciones sistemáticas con el fin de identificar posibles mejoras.

Tabla 2. Indicador: Diseño del proceso

\begin{tabular}{llcc}
\hline \multicolumn{1}{c}{ Ítem } & $\square$ & Categorías \\
\hline 4. $\begin{array}{l}\text { Los procesos son diseñados para obtener resultados de } \\
\text { bajo costo. }\end{array}$ & 4,34 & Muy alta aplicación \\
$\begin{array}{l}\text { 5. } \\
\text { Invierten en rediseñar sus procesos para optimizar los } \\
\text { recursos. }\end{array}$ & 4,00 & Alta aplicación \\
$\begin{array}{l}\text { 6uentan con procedimientos por escrito sobre los } \\
\text { procesos, cuya información es conocida por todos los } \\
\text { interesados }\end{array}$ & 3,76 & Alta aplicación \\
\hline
\end{tabular}

Fuente: Elaboración propia.

Los resultados obtenidos permiten validar la teoría expuesta por Arnoletto (2010), quien refiere que las herramientas para diseñar los procesos son maneras sencillas de dar sentido a lo que sucede en un proceso. De manera que, a criterio de la investigadora, las universidades públicas analizadas definen y seleccionan los pasos que llevan a cabo para cumplir los procesos de admisión, tratando de minimizar tiempos y maximizar beneficios.

Con respecto al indicador control del proceso, este presenta una media de 3,71 , tal como se evidencia en la Tabla 3, la cual indica alta aplicación del indicador como parte del proceso operativo aplicado en los departamentos de admisión estudiantil de las universidades públicas de la Costa Oriental del Lago, a fin de garantizar que todo se haga conforme fue planeado y organizado, según las órdenes dadas, para identificar los errores o desvíos con el fin de corregirlos y evitar su repetición.

Tabla 3. Indicador: Control del proceso

\begin{tabular}{|c|c|c|}
\hline Ítem & $\square$ & Categorías \\
\hline $\begin{array}{l}\text { 7. Evalúan el flujo de trabajo de las diferentes áreas de } \\
\text { producción, a través de indicadores. }\end{array}$ & 3,72 & Alta aplicación \\
\hline $\begin{array}{l}\text { 8. Los procesos comprenden sistemas flexibles con fácil } \\
\text { manejo. }\end{array}$ & 3,62 & Alta aplicación \\
\hline $\begin{array}{l}\text { 9. Llevan a cabo auditorías internas para dar seguimiento } \\
\text { a sus procesos. }\end{array}$ & 3,79 & Alta aplicación \\
\hline Total indicador: & 3,71 & Alta aplicación \\
\hline
\end{tabular}

Fuente: Elaboración propia. 
Para la investigadora estos resultados reflejan que las instituciones analizadas recolectan datos para realizarles mejoras, buscando elevar la calidad del proceso y aumentar la eficiencia con reducción de costos. Dado los resultados mostrados, se evidencia alta congruencia con lo planteado por Ruiz (2013), para quien el control es la consecuencia lógica de administrar, una función indelegable de todo cargo con responsabilidad.
En la Tabla 4 se observa el resultado para el indicador mejoras del proceso, con una media de 3,71 correspondiente a una alta aplicación, refiriendo con ello que en los departamentos de admisión estudiantil se busca optimizar los distintos procesos administrativos ejecutados por las instituciones analizadas, a partir de lograr un entendimiento integral de los mismos por parte del grupo de trabajo.

Tabla 4. Indicador: Mejoras del proceso

\begin{tabular}{lll}
\hline \multicolumn{1}{c}{ Ítem } & $\square$ & Categorías \\
\hline $\begin{array}{l}\text { 10. Los gerentes ejercen un liderazgo que propicia el } \\
\text { desempeño óptimo de los empleados }\end{array}$ & 4,21 & Muy alta aplicación \\
$\begin{array}{l}\text { 11. El director del departamento da a conocer al trabajador } \\
\text { su papel dentro del proceso laboral. }\end{array}$ & 3,17 & Moderada aplicación \\
$\begin{array}{l}\text { 12. Fijan objetivos de satisfacción al usuario, para conducir } \\
\text { la ejecución de los procesos. }\end{array}$ & 3,76 & Alta aplicación \\
\hline Total indicador: & $\mathbf{3 , 7 1}$ & Alta aplicación
\end{tabular}

Fuente: Elaboración propia.

Visto desde la perspectiva de los resultados, para la investigadora, la mejora de los procesos en los departamentos de admisión estudiantil, se desarrollan con alta aplicación, esto debido a que el personal que labora en las universidades analizadas se esfuerza y comprometen en realizar un buen trabajo. Lo mostrado coincide de muy alta manera con lo expuesto por Heizer y Render (2009), para quienes solo puede existir mejora de procesos si los integrantes de la organización se esfuerzan y comprometen en hacer el trabajo bien, para lo que se necesita asignar responsables que se encarguen de los procesos.

En lo concerniente al indicador selección del proceso, se observa en la Tabla 5 una media de 3,54, indicando alta aplicación en la selección de las actividades necesaria para los procesos de admisión, que permitan la satisfacción total de los usuarios. Es evidente, de acuerdo a lo mostrado, que en los departamentos de admisión estudiantil de las universidades públicas de la Costa Oriental del Lago, se identifican los procesos críticos en los que se requiere sobresalir con excelencia, satisfaciendo los objetivos. 
Tabla 5. Indicador: Selección del proceso

\begin{tabular}{|c|c|c|}
\hline Ítem & $\square$ & Categorías \\
\hline 13. Realizan estudios de tiempos en las líneas de atención. & 3,86 & Alta aplicación \\
\hline 14. Eliminan los procesos que restan valor al servicio. & 3,66 & Alta aplicación \\
\hline $\begin{array}{l}\text { 15. Cumplen con las fechas de corrección en desviaciones } \\
\text { encontradas durante la auditoría interna. }\end{array}$ & 3,10 & Moderada aplicación \\
\hline Total indicador: & 3,54 & Alta aplicación \\
\hline
\end{tabular}

Fuente: Elaboración propia.

Los resultados validan, en muy alta medida, a Kaplan y Norton (2009), para quienes se deben analizar los procesos internos, en aras de conseguir la satisfacción total del cliente y altos niveles de rendimiento financiero, con la finalidad de determinar las áreas críticas y aplicar los correctivos necesarios para detectar la selección de procesos que mejor se adapte a la actividad.

De igual manera, a criterio de la investigadora la selección del proceso forma parte del proceso operativo aplicado en los departamentos de admisión estudiantil de las universidades públicas de la Costa Oriental del Lago, a fin de determinar las orientaciones necesarias y las acciones pertinentes para obtener un sistema que, atendiendo a la misión, la visión, los valores $\mathrm{y}$ los planes de desarrollo de cada institución, dé una respuesta socialmente justa a la demanda de ingreso de los aspirantes a sus aulas.

Ya analizados cada uno de los indicadores considerados en la dimensión denominada procesos operativos, se pasa a verificar los resultados arrojados para esta dimensión. En la Tabla 6 se observa un promedio de 3,83 indicando alta aplicación de los planes de acción para alcanzar los objetivos operativos y conseguir la misión de los departamentos de admisión estudiantil en conjunto y de esa forma crear una ventaja competitiva para las universidades públicas de la Costa Oriental del Lago.

Tabla 6. Dimensión: Procesos operativos

\begin{tabular}{lcl}
\hline \multicolumn{1}{c}{ Indicador } & $\square$ & Categorías \\
\hline Decisión del Proceso & 4,17 & Alta Aplicación \\
Diseño del Proceso & 4,03 & Alta Aplicación \\
Control del Proceso & 3,71 & Alta Aplicación \\
Mejoras del Proceso & 3,71 & Alta Aplicación \\
Selección del Proceso & 3,54 & Alta Aplicación \\
\hline Total dimensión: & $\mathbf{3 , 8 3}$ & Alta Aplicación \\
\hline
\end{tabular}

Fuente: Elaboración propia.

Los resultados de alta aplicación de la dimensión procesos operativos validan lo expuesto por Rodríguez (2008), para quien en este proceso se establecen criterios y métodos que aseguren de manera eficaz las actividades que comprenden, lo que involucra, centrarse en actividades operacionales y en las características relevantes que permitan el control y la gestión del proceso. 
Con base a estas afirmaciones del autor citado, se deduce que las universidades públicas estudiadas, dado que se tiene alta aplicación de los procesos operativos, se posicionan con una fortaleza en el aseguramiento de la gestión realizada en los procesos de admisión. Esto es coincidente con el criterio de la investigadora, cuando afirma que a través de este proceso se organizan los esfuerzos y la utilización de los recursos para lograr la satisfacción de aquello que está vinculado con los procesos que definen al sistema organizacional y son los responsables en añadir el valor agregado en el servicio prestado.

\section{CONCLUSIONES}

Con relación al proceso de operaciones aplicada en los departamentos de admisión estudiantil de las universidades públicas de la Costa Oriental del Lago, se concluye que se aplican planes de acciones que permiten planificar y controlar los servicios ofrecidos para alcanzar los objetivos operativos acorde a la misión de la institución en conjunto y de esa forma crear una ventaja competitiva, a través de la decisión del proceso, el diseño del proceso, control del proceso, mejoras del proceso y la selección del mismo.

En definitiva, se observó que se analizan y optimizan la interacción de los procesos para obtener las metas deseadas, apoyada en los diseños de procesos, a fin de garantizar que todo se haga conforme fue planeado y organizado, en la búsqueda de optimizar los distintos procesos administrativos ejecutados, analizando los procesos internos para conseguir la satisfacción total de los usuarios.
REFERENCIAS

Arnoletto, E. (2010). Gestión organizacional en los gobiernos locales. Recuperado de http://www.eumed.net/librosgratis $/ 2010 \mathrm{~d} / 777$

Bravo, J. (2011). Gestión de procesos con responsabilidad social. Santiago de Chile: Editorial Evolución, S.A

Cervera, J. (2002). La Transición a las nuevas ISO $9000-2000$ y su implantación: Un Plan sencillo y práctico con ejemplos. Madrid: Ediciones Díaz de Santos

Chase, R.; Aquilano, N. Y Jacobs, R. (2009). Administración de operaciones "producción y cadena de suministros". (12 ${ }^{\circ}$ ed.). México: Editorial McGrawHill-Irwin

Fernández, A. (2010). Gestión por Procesos. 4ta. Edición. Madrid: Editorial Esic

Galván, P. (2009). Mejora continua en la gestión por procesos. Vigo: Editorial Ideas propias

Guevara, M.; Terán, I. y Rodríguez, Z. (2011). La admisión y el rendimiento académico en los estudios de medicina. Escuela de Medicina "Dr. Witremundo Torrealba". Universidad de Carabobo, Venezuela. Revista Comunidad y salud. Vol. 9. Nro. 2. Diciembre pp. 34-43. Recuperado de www.scielo.org.ar

Heizer, J. y Render, B. (2009). Dirección de la producción y de operaciones. Decisiones estratégicas. México: Editorial Pearson Prentice Hall

Kaplan, R. y Norton, D. (2009). Cómo utilizar el cuadro de mando integral para implantar y gestionar su estrategia. Segunda Edición. Madrid: Ediciones Gestión 2000

Krajewski, L. y Ritzman, L. (2008). Administración de operaciones: estrategia 
y análisis. 7ma edición. México: Editorial Pearson Educación, S.A

Mallar, M. (2010). La gestión por procesos: un enfoque de gestión eficiente. Revista visión de futuro. Año 7. Vol. 13. Nro. 1 enero-junio S/N de pág. Recuperado de www.scielo.org.ar

Mariño, H. (2001). Gerencia de procesos. Bogotá: Alfaomega

Mariño, H. (2001). Gerencia de procesos. Bogotá: Alfaomega

Pérez, J. (2010). Gestión por procesos. Madrid: Editor ESIC Editorial

Rey, D. (2013). Todo sobre la gestión por procesos. Lecciones aprendidas. Sinapsys Business Solutions. Recuperado de www.sinap-sys.com

Rodríguez, J. (2008). Decisiones Gerenciales Efectivas. Fundamentos para la solución de problemas administrativos. México: Editorial Trillas
Rojas, J. (2003). Gestión por procesos y atención al usuario en los establecimientos del Sistema Nacional de Salud. Recuperado de www.eumed.net/libros/2007a/

Ruiz, M. (2013). Gestionar los procesos. Guía de aplicación a las empresas. Madrid: Editorial Díaz de Santos. Colección Monografías. Serie Administración y Marketing

Serrano, J. (2003). Psicología de la instrucción, Vol. I: Historia, Concepto, Objeto y Método. Murcia: D M Editor

Skinner, W. (2010). Administración estratégica de operaciones. México: McGraw-Hill

Slack, N., Chambers, S., y Johnston, R. (2007). Gerencia de Operaciones. 6ta edición. México: Editorial Mc GrawHill 